\title{
Effect of Clostridium butyricum supplementation on the development of intestinal flora and the immune system of neonatal mice
}

\author{
RUI-XUE MIAO ${ }^{1,2}$, XIN-XIN ZHU $^{3}$, CHAO-MIN WAN ${ }^{1,2}$, ZHI-LING WANG $^{1,2}$, YANG WEN $^{1,2}$ and YI-YUAN LI ${ }^{1,2}$ \\ ${ }^{1}$ Department of Pediatrics, West China Second Hospital, Sichuan University; ${ }^{2}$ Key Laboratory of \\ Birth Defects and Related Diseases of Women and Children, Ministry of Education, Sichuan University, Chengdu, \\ Sichuan 610041; ${ }^{3}$ Department of Pediatrics, Xi'an Children's Hospital, Xi'an, Shanxi 710000, P.R. China
}

Received May 10, 2016; Accepted March 24, 2017

DOI: 10.3892/etm.2017.5461

\begin{abstract}
The objective of the present study was to examine whether Clostridium butyricum supplementation has a role in the regulation of the intestinal flora and the development of the immune system of neonatal mice. A total of 30 pregnant BALB/c mice, including their offspring, were randomly divided into three groups: In the maternal intervention group (Ba), maternal mice were treated with Clostridium butyricum from birth until weaning at postnatal day 21 (PD21) followed by administration of saline to the offspring at PD21-28; in the offspring intervention group $(\mathrm{Ab})$, breast-feeding maternal mice were supplemented with saline and offspring were directly supplemented with Clostridium butyricum from PD21-28; in the both maternal and offspring intervention group $(\mathrm{Bb})$, both maternal mice and offspring were supplemented with Clostridium butyricum at PD 0-21 and at PD21-28. While mice in the control group were given the same volume of normal saline. Stool samples from the offspring were collected at PD14, -21 and -28 to observe the intestinal flora by colony counts of Enterococcus spp., Enterobacter spp., Bifidobacterium spp. and Lactobacillus spp. Detection of intestinal secreted immunoglobulin A (sIgA) levels and serum cytokine (interferon- $\gamma$, and interleukin-12, -4 and -10) levels in offspring was performed to evaluate the effect on their immune system. The results revealed that compared with the control group, offspring in the Ba group displayed significantly decreased stool colony counts of Enterococcus spp. $(\mathrm{t}=3.123, \mathrm{P}<0.01)$ at $\mathrm{PD} 14$ and significantly decreased counts of Enterobacter spp. at PD14 and $-21(\mathrm{t}=2.563, \mathrm{P}<0.05$ and $\mathrm{t}=2.292, \mathrm{P}<0.05$, respectively). Compared with the
\end{abstract}

Correspondence to: Dr Chao-Min Wan, Department of Pediatrics, West China Second Hospital, Sichuan University, 20 3rd Section of Renmin South Road, Chengdu, Sichuan 610041, P.R. China E-mail:wcm0220@126.com

Key words: probiotics, Clostridium butyricum, intestinal flora, immune system control group, the stool colony counts of Bifidobacterium spp. and Lactobacillus spp. were significantly increased in the $\mathrm{Ba}$ group at $\mathrm{PD} 21(\mathrm{t}=3.085, \mathrm{P}<0.01$ and $\mathrm{t}=2.8508, \mathrm{P}<0.05$, respectively). The $\mathrm{Ab}$ group had significantly higher stool colony counts of Bifidobacterium spp. and Lactobacillus spp. at $\mathrm{PD} 28$, compared with the control group $(\mathrm{Q}=7.679, \mathrm{P}<0.01$ and $\mathrm{Q}=6.149, \mathrm{P}<0.01$, respectively). There were no significant differences identified in the sIgA levels of the intestinal fluid and serum cytokine levels between the control group and the intervention groups. In conclusion, Clostridium butyricum administered to breast-feeding maternal mice was able to regulate the intestinal flora balance in their offspring. However, due to insignificant effects on sIgA level and the associated cytokines, Clostridium butyricum had a limited influence on the balance of type 1 vs. type 2 T-helper cells. However, using Clostridium butyricum as an invention may be a safe method for improving the balance of intestinal flora and associated processes in offspring.

\section{Introduction}

Intestinal microbiota ( $\sim 10^{14}$ microbial cells/person), contribute to human health and are known as the most important and complex ecosystem (1). After birth, the composition of gut microbiota transforms gradually from aerobic and facultative anaerobic bacteria into predominantly obligate anaerobes. Therefore, early life is the most significant period for the structural transformation of the intestinal flora profile. In principle, the microbial ecosystem reaches a profile characteristic of adult microbiota in composition and diversity by the end of the first 3-5 years of life with changes in parameters such as $\mathrm{pH}$, oxygen content and nutritional consumption (2).

Microbial colonization has a crucial role not only in the host's acquisition of nutrients and modulation of metabolism but also in promoting immune system development and maturation. As a portion of the mucosal barrier, microbiota are helpful in the protection against intestinal epithelial injury and boost the immune system to mature in the growth of mucosa-associated lymphoid tissue, the amount of secreted immunoglobulin A (sIgA), regulation of cytokines and expansion of T-cell populations. T-helper cells (Th) have 
a critical role in amplifying immune responses with Th1 and Th2 cells driving 'cellular immunity' and 'humoral immunity' of the adaptive immunity system. Th-cell populations may be classified by the cytokines they secrete. Th1 cells are heavily reliant on tumor necrosis factor- $\gamma$ (IFN- $\gamma$ ) and interleukin-12 (IL-12), while Th2 cells are characterized by the cytokines IL-4 and IL-10 $(3,4)$.

Except for the interaction between gut microbiota and the host, which is reflected in the physiological function, it has long been considered that disorders of the microbial system have a direct or indirect association with various diseases (intestinal as well as extra-intestinal), including irritable bowel syndrome, inflammatory bowel disease, antibiotic-associated diarrhea (AAD), autoimmune diseases and even central nervous system disorders $(5,6)$. In children and infants with chronic diarrhea and allergic disease, a decrease in Lactobacillus spp. and Bifidobacterium spp. is frequently observed (7).

Probiotics, live microorganisms that confer a health benefit on the host when administered clinically to inhabit inflammatory reactions, abrogate disorders of the intestinal flora (8), prevent and treat diarrhea and allergy (9) and alleviate symptoms of patients with ulcerative colitis (10). However, different species or even different strains of the same species may have different functions in the regulation of intestinal flora and development of the intestinal immune system (11). Clostridium butyricum has the capacity to produce large amounts of short-chain fatty acids, which promote intestinal absorption of water and sodium $(12,13)$. Studies have found that Clostridium butyricum has a regulatory effect in the intestinal flora by increasing the amount of Lactobacillus spp (14) and preventing AAD (15). The present study was performed to explore the influence of Clostridium butyricum [China General Microbiological Culture Collection Center (CGMCC) no. 0313-1] on the regulation of gut microflora by assessing the colony counts of Enterococcus spp., Enterobacter spp., Bifidobacterium spp. and Lactobacillus spp. and on the development of the immune system by detecting intestinal $\operatorname{sIg}$ A and serum cytokines IFN- $\gamma$, IL-12, IL-4 and IL-10.

\section{Materials and methods}

Animals and reagents. A total of 30 female, pregnant BALB/c mice (age, 8 weeks; weight, 25-30 g; one day following pregnancy) were obtained from the Laboratory Animal Center of Sichuan University. The animal experiment was approved by the Ethics Committee of Sichuan University. Clostridium butyricum (CGMCC no. 0313-1) was provided by Beijing Eastern Baixin Biotechnology Co., Ltd. (Beijing, China) and was diluted to a concentration of $5 \times 10^{8}$ colony-forming units $(\mathrm{CFU}) / 0.5 \mathrm{ml}$ with normal saline. Azide oxygen agar medium, Eosion Methylene Blue agar medium (EMB), Lactobacillus selection medium (LBS) and Bifidobacterium medium (BBL) were obtained from LuQiao Biotechnology Company (Taizhou, China) and were used to culture Enterobacter spp., Enterococcus spp., Lactobacillus spp. and Bifidobacterium spp., respectively. All media were sterilized at high pressure at $115^{\circ} \mathrm{C}$ for $20 \mathrm{~min}$. The $\operatorname{sig} \mathrm{A}$, IL-12, IL-4, IL-10 and IFN- $\gamma$ ELISA kits were purchased from R\&D Systems, Inc. (Minneapolis, MN, USA). Pilocarpine was obtained from Shanghai Yuanye Biotechnology Co., Ltd., (Shanghai, China).

Experimental design. The influence of Clostridium butyricum on the regulation of gut microflora and immune system development was assessed by observing and analyzing the level of four bacterial faces (Enterococcus spp., Enterobacterium spp., Lactobacillus spp. and Bifidobacterium spp.), the amount of intestinal sIgA, cytokines IFN- $\gamma$, IL-12 as biomarkers of Th1 cells and IL-4 and IL-10 as markers of Th2 cells in offspring. The day of birth was defined as postnatal day 1 (PD1). The mice were weaned off on PD21, which is the natural time of weaning.

The experimental design is illustrated in Fig. 1. The thirty maternal mice and their offspring were randomly divided into three groups following successful delivery ( $\mathrm{n}=10$ mouse pups per group): The control group (Aa), offspring intervention group $(\mathrm{Ab})$, maternal intervention group $(\mathrm{Ba})$ and maternal and offspring intervention group $(\mathrm{Bb})$. In the $\mathrm{Ba}$ group, maternal mice were treated with Clostridium butyricum by gavage at a dosage of $5 \times 10^{8} \mathrm{CFU}$ per day during lactation (from PD1 to PD20) and offspring were given $0.5 \mathrm{ml}$ normal saline by gavage per day after weaning (from PD21 to PD28). In the $\mathrm{Ab}$ group, maternal mice were given $0.5 \mathrm{ml}$ normal saline per day by gavage during lactation and offspring were treated with Clostridium butyricum by gavage at a dosage of $5 \times 10^{8} \mathrm{CFU}$ per day after weaning. In the maternal and offspring intervention group $(\mathrm{Bb})$, both maternal mice and offspring were supplemented with Clostridium butyricum at PD0-21 and at PD21-28. In the control group, maternal mice were given $0.5 \mathrm{ml}$ normal saline per day by gavage during lactation and offspring were administered the same amount of saline after weaning. Other environmental factors and diet were kept consistent among the groups. The mice were maintained in a $12 \mathrm{~h}$ light/dark cycle at a temperature of $20-25^{\circ} \mathrm{C}$ in $40-70 \%$ humidity. They were given $1-3 \mathrm{~g} / \mathrm{day}$ food and 4-7 ml/day water each. On PD14, -21 and -28, stool specimens were collected from the offspring and aliquots were individually inoculated into the four media mentioned above. Samples in Azide oxygen agar medium and EMB medium were placed in an incubator at $37^{\circ} \mathrm{C}$ and those in LBS and BBL media were placed into an anaerobic box for bacterial culture at $37^{\circ} \mathrm{C}$. Finally, bacteria were quantified after $48 \mathrm{~h}$. Similarly, sIgA in the intestinal fluid and serum cytokine (IL-12, IL-4, IL-10 and IFN- $\gamma$ ) levels were detected using the corresponding ELISA kits at PD14, -21 and -28. A total of $0.2 \mathrm{ml}$ serum was obtained from blood obtained from the inner canthal vein. To determine $\operatorname{sgA}$, intestinal fluid was gathered by intraperitoneal injection of $10 \mathrm{mg} / \mathrm{kg}$ pilocarpine after administration of an enema without anesthesia as previously described (16).

Statistical analysis. The numbers of trials between two groups were analyzed by using an independent-samples t test and the differences among multiple groups were analyzed by one-way analysis of variance (ANOVA). A Student-Newman-Keul-Q post hoc test was then used for individual comparisons when the ANOVA analysis indicated a significant difference among groups. SPSS, version 18.0 (SPSS, Inc., Chicago, IL, USA) was used. Values are expressed as the mean \pm standard deviation. 


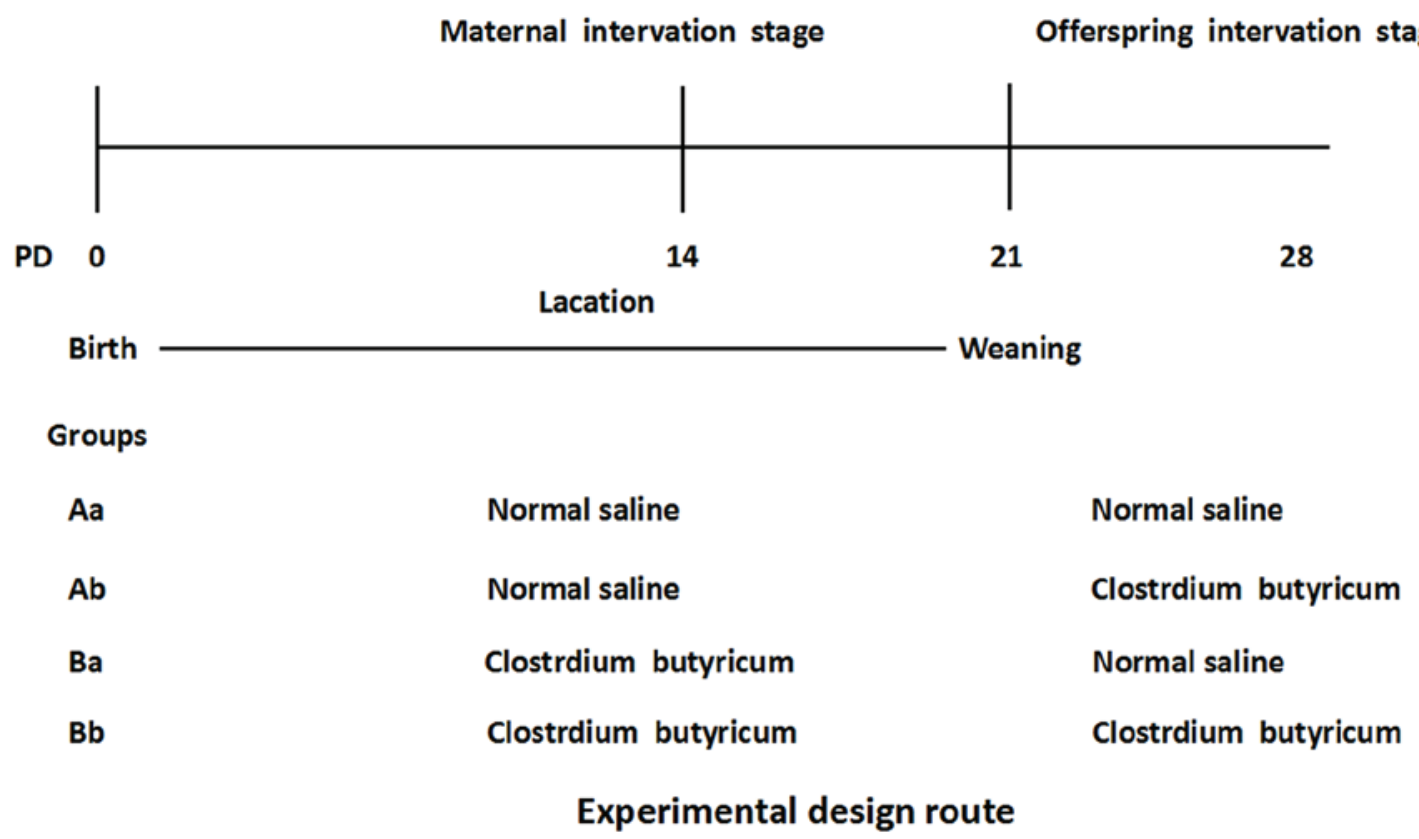

Figure 1. Experimental design. The experiment included two stages: Maternal mice received Clostridium butyricum supplementation by gavage during lactation and offspring mice received intervention after weaning. The experiment was divided into three groups according to different modes of administration of Clostridium butyricum or treatment with normal saline. Aa, control group; Ba, maternal intervention group; Ab, offspring intervention group; Bb, maternal and offspring intervention group; PD, postnatal days.

$\mathrm{P}<0.05$ was considered to indicate a statistically significant difference.

\section{Results}

Clostridium butyricum supplementation affects the intestinal flora in mouse pups. To investigate the role of Clostridium butyricum in modulating the gut flora, four common bacterial strains of the intestine, Enterococcus spp., Enterobacter spp., Lactobacillus spp. and Bifidobacterium spp., were assessed. As revealed in Fig. 2, supplementation of breast-feeding maternal mice with Clostridium butyricum (Ba group) significantly decreased the quantity of intestinal Enterococcus spp. $(\mathrm{t}=3.123, \mathrm{P}<0.01)$ and Enterobacter $\mathrm{spp}$. $(\mathrm{t}=2.563, \mathrm{P}<0.05)$ at PD14 in the offspring compared with that in the control group. At PD21 the count of intestinal Enterococcus spp. in the offspring of the Ba group was further significantly decreased $(\mathrm{t}=2.292, \mathrm{P}<0.05)$ compared with the Aa control group, while the count of Enterobacter spp. was not ( $\mathrm{t}=1.297$, P>0.05; Fig. 3). Additionally, maternal supplementation significantly increased the quantity of intestinal Lactobacillus spp. $(\mathrm{t}=2.8508, \mathrm{P}<0.01)$ and Bifidobacterium spp. ( $\mathrm{t}=3.085, \mathrm{P}<0.01)$ in the offspring of the Ba group at PD21 compared with that in the control group, while it had no significant effect at PD14 $(\mathrm{t}=1.213, \mathrm{P}>0.05$ and $\mathrm{t}=0.414$, $\mathrm{P}>0.05$ respectively). Following ablactation, Bifidobacterium spp. and Lactobacillus spp. were significantly increased in the $\mathrm{Ab}$ group compared with the control group at PD28 $(\mathrm{Q}=7.679, \mathrm{P}<0.01$ and $\mathrm{Q}=6.149, \mathrm{P}<0.01$, respectively), however no significant differences were observed in the quantity of Enterococcus spp. $(\mathrm{Q}=0.973, \mathrm{P}>0.05)$ and Enterobacter $\mathrm{spp}$. $(\mathrm{Q}=1.757, \mathrm{P}>0.05)$ compared with the control at the same time point (Fig. 4). Bifidobacterium spp. and Lactobacillus spp. were also significantly increased in the Ab group compared with the $\mathrm{Ba}$ and $\mathrm{Bb}$ group $(\mathrm{P}<0.01)$. These results suggest that the role of breast milk on the diet of the offspring requires further exploration.

Clostridium butyricum supplementation does not affect the intestinal secretion of sIgA in mouse pups. Next, the effects of Clostridium butyricum on intestinal sIgA in mouse pups were assessed. No statistically significant differences were observed between the intervention groups and the control group at PD14 $(\mathrm{t}=-0.737, \mathrm{P}>0.05$; Fig. 5), $-21(\mathrm{t}=0.494, \mathrm{P}>0.05$; Fig. 5$)$ and -28 $\left(\mathrm{Q}_{\mathrm{Ba}}=0.380, \mathrm{P}>0.05 ; \mathrm{Q}_{\mathrm{Ab}}=3.564, \mathrm{P}>0.05 ; \mathrm{Q}_{\mathrm{Bb}}=3.461, \mathrm{P}>0.05\right.$; Fig. 6).

Effect of Clostridium butyricum on cytokine secretion in offspring. To examine the effects of Clostridium butyricum supplementation on developing immunity, circulating IL-12 and IFN- $\gamma$ were assessed as biomarkers for Th1 cells, while IL-4 and IL-10 served as biomarkers for Th2 cells. As revealed in Table I, a slight but insignificant increase in the secretion of IL-12 and IFN- $\gamma$ was detected in the Ba group at PD21 compared with the control group. Similarly, no significant differences were observed in the secretion of IL-4 and IL-10 at PD21 compared with the control group. The Ab group also demonstrated a slight but insignificant increase in IL-12 and IFN- $\gamma$ at PD28 compared with the control group.

\section{Discussion}

The intestine is an important organ that maintains the immunity and nutrition of the body, and it is also the biggest bacterial storeroom in the body. During infancy, the intestinal flora is not stable. In the present study, supplementation with Clostridium butyrium reduced the colony count of Enterobacter spp. and Enterococcus spp. and increased the colony count of 


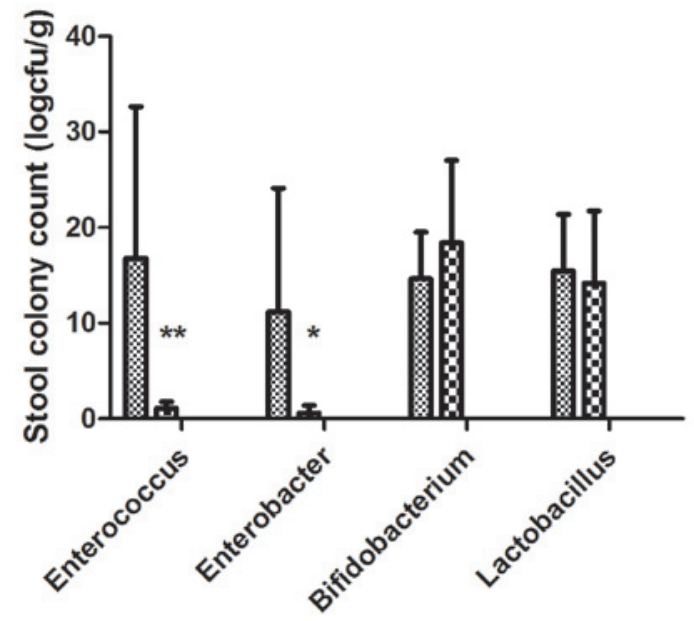

Figure 2. Colony count of four bacteria strains at PD14 in the intestines of mice offspring following maternal intervention. The intestinal levels of Enterococcus spp. $(\mathrm{P}<0.01)$ and Enterobacter spp. $(\mathrm{P}<0.05)$ were significantly decreased at PD14 and those of Lactobacillus spp. $(\mathrm{P}>0.05)$ and Bifidobacterium spp. $(\mathrm{P}>0.05)$ were not statistically different at PD14 compared with the control group. All values are expressed as the mean \pm standard deviation. ${ }^{*} \mathrm{P}<0.05$ and ${ }^{* *} \mathrm{P}<0.01$ vs. Aa. Aa, control group; $\mathrm{Ba}$, maternal intervention group; $\mathrm{PD}$, postnatal days; cfu, colony-forming units.

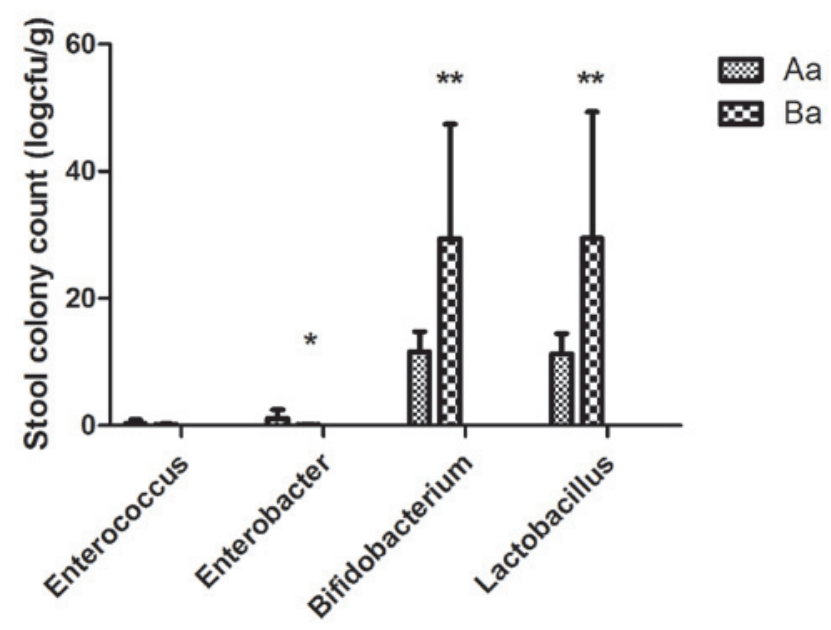

Figure 3. Colony count of four bacteria strains at PD21 in the intestines of mice offspring following maternal intervention. The intestinal levels of Enterobacter spp. $(\mathrm{P}<0.05)$, Bifidobacterium spp. $(\mathrm{P}<0.01)$ and Lactobacillus spp. $(\mathrm{P}<0.05)$ were significantly increased at $\mathrm{PD} 21$ and the level of Enterococcus spp. (P>0.05) was not statistically different compared with the control group. All values are expressed as the mean \pm standard deviation ${ }^{*} \mathrm{P}<0.05$ and ${ }^{* *} \mathrm{P}<0.01$ vs. Aa. Aa, control group; Ba, maternal intervention group; $\mathrm{PD}$, postnatal days; cfu, colony-forming units.

Lactobacillus spp. and Bifidobacterium spp in the stool of the offspring. Moreover, when the maternal mice were treated with Clostridium butyrium by gavage, the decrease in intestinal Enterobacter spp. and Enterococcus spp. at PD14 in the offspring occurred earlier than the increase of Lactobacillus spp. and Bifidobacterium spp. at PD21, which was in line with the process of gut flora colonization. After birth, the intestinal microbiota is dominated by aerobic and facultative anaerobic bacteria such as Enterobacter spp. and Enterococcus spp., which are then replaced by a complex anaerobe-dominated flora, including Lactobacillus spp. and Bifidobacterium

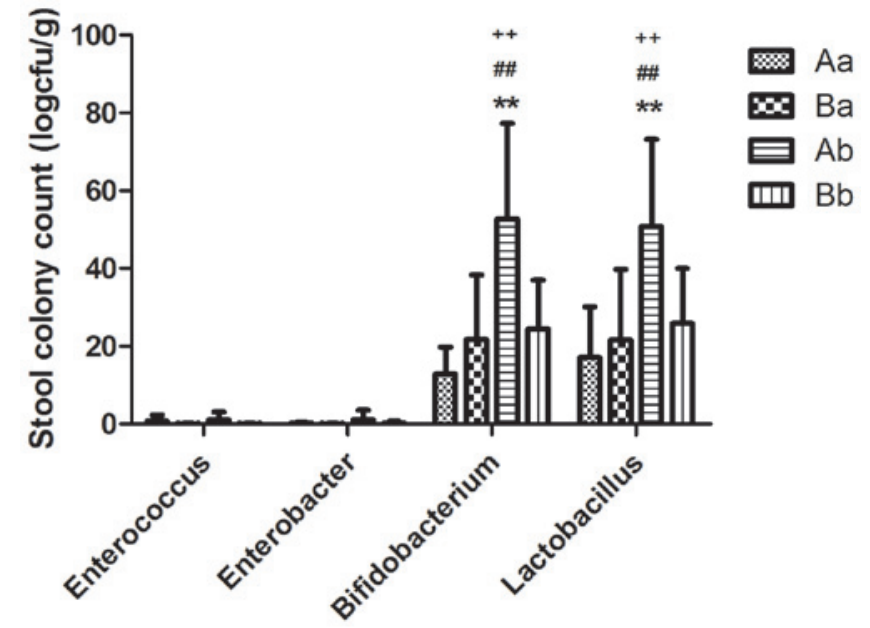

Figure 4. Colony counts of four bacterial strains in the intestines of mice offspring in the three groups at PD28. Following ablactation, Bifidobacterium spp. $(\mathrm{P}<0.01)$ and Lactobacillus spp. $(\mathrm{P}<0.01)$ were significantly increased in the $\mathrm{Ab}$ group compared with the Aa group. Significant differences were also observed between the levels of Bifidobacterium spp. and Lactobacillus spp. in the $\mathrm{Ab}$ and other groups $(\mathrm{P}<0.01)$. All values are expressed as the mean \pm standard deviation. ${ }^{* *} \mathrm{P}<0.01$ vs. $\mathrm{Aa} ;{ }^{\# \#} \mathrm{P}<0.01$ vs. $\mathrm{Ba} ;{ }^{++} \mathrm{P}<0.01$ vs. $\mathrm{Bb}$. Aa, control group; $\mathrm{Ba}$, maternal intervention group; $\mathrm{Ab}$, offspring intervention group; $\mathrm{Bb}$, maternal and offspring intervention group; cfu, colony-forming units.

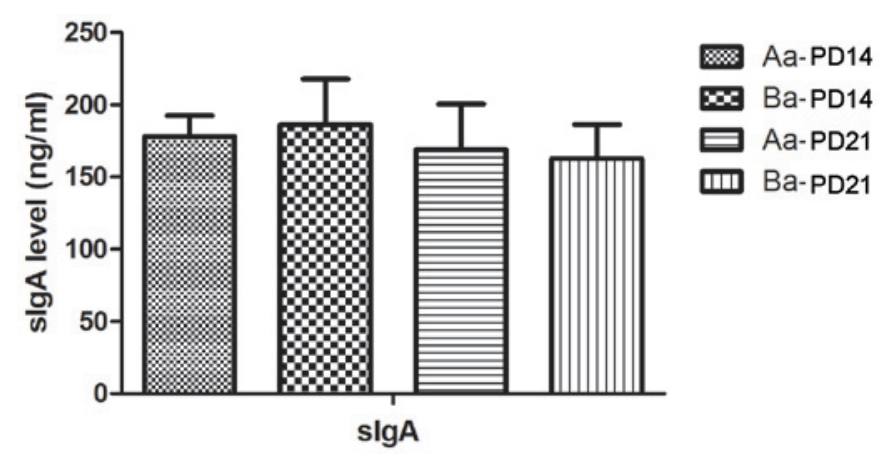

Figure 5. sIgA levels in the intestinal fluid of offspring at PD14 and PD21 following maternal intervention with Clostridium butyricum. No significant differences in offspring intestinal juice $\operatorname{SIgA}$ were detected between the Ba group at PD14 and PD21 and the control group $(\mathrm{P}>0.05)$. All values are expressed as the mean \pm standard deviation. Aa, control group; Ba, maternal intervention group; PD, postnatal days; sIgA, secreted immunoglobulin A.

spp., an anaerobe-dominated flora that constitutes a portion of the mucosal barrier to protect against pathogen invasion and maintain the balance of microbiota. Over the first few weeks of life, changes in $\mathrm{pH}$ and oxygen content and nutritional consumption occur, which are also affected by feeding patterns, including breast or formula feeding. In the present study, the composition and quantity of bacterial strains in the intestinal flora of neonatal mice changed when the maternal mice were treated with Clostridium butyrium during lactation. The above results demonstrated that Clostridium butyrium affects offspring through breast milk, while having a direct effect when orally administered after weaning. This is in accordance with the findings of a previous study on breast-fed subjects, which reported that infants were able to acquire probiotics via breast milk (17). Clostridium butyrium does not colonize in the normal intestinal flora and keeps a balance of 
Table I. Cytokine expression in offspring after Clostridium butyricum supplementation.

\begin{tabular}{llllll}
\hline Group & Time-point & IFN- $\gamma(\mathrm{pg} / \mathrm{ml})$ & IL-12 $(\mathrm{pg} / \mathrm{ml})$ & IL-4 $(\mathrm{pg} / \mathrm{ml})$ & $\mathrm{IL}-10(\mathrm{pg} / \mathrm{ml})$ \\
\hline $\mathrm{Aa}$ & PD 14 & $273.84 \pm 125.12$ & $222.52 \pm 30.08$ & $65.292 \pm 27.60$ & $86.172 \pm 8.61$ \\
$\mathrm{Ba}$ & PD 14 & $286.85 \pm 86.85$ & $193.90 \pm 29.55$ & $62.780 \pm 14.57$ & $71.130 \pm 11.07$ \\
$\mathrm{Aa}$ & PD 21 & $262.87 \pm 62.87$ & $219.27 \pm 19.27$ & $65.80 \pm 65.80$ & $87.35 \pm 17.43$ \\
$\mathrm{Ba}$ & PD 21 & $362.15 \pm 62.15$ & $327.97 \pm 27.97$ & $73.06 \pm 3.067$ & $88.32 \pm 8.327$ \\
$\mathrm{Aa}$ & PD 28 & $269.05 \pm 69.05$ & $204.30 \pm 04.30$ & $43.82 \pm 3.82$ & $77.08 \pm 7.08$ \\
$\mathrm{Ba}$ & PD 28 & $284.11 \pm 84.11$ & $203.65 \pm 03.65$ & $57.51 \pm 7.515$ & $74.29 \pm 4.295$ \\
$\mathrm{Ab}$ & PD 28 & $334.70 \pm 34.70$ & $235.97 \pm 35.97$ & $56.08 \pm 6.087$ & $68.58 \pm 8.58$ \\
$\mathrm{Bb}$ & PD 28 & $312.63 \pm 211.63$ & $258.38 \pm 52.70$ & $57.06 \pm 14.3$ & $67.49 \pm 11.80$
\end{tabular}

All values are presented as the mean \pm standard deviation. There were no statistically significant differences among groups. IFN, interferon; IL, interleukin; Aa, control group; Ba, maternal intervention group; $\mathrm{b}$, offspring intervention group; $\mathrm{Bb}$, maternal and offspring intervention group; PD, postnatal days.

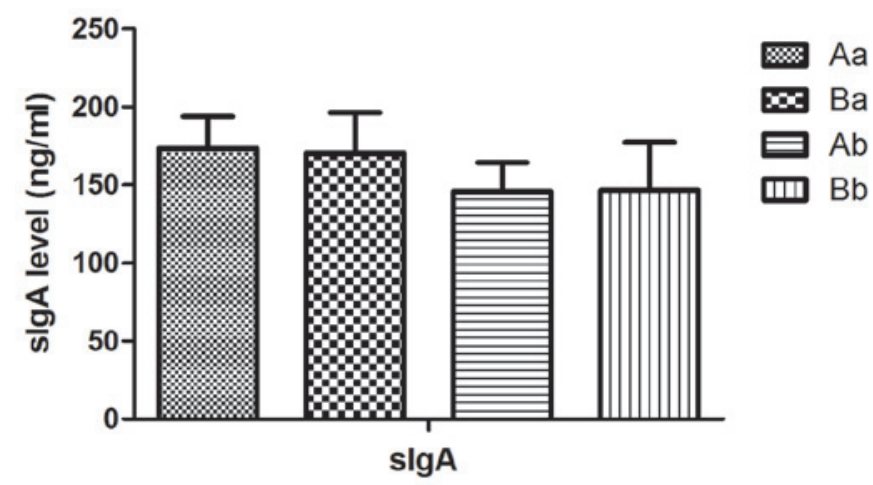

Figure 6. sIgA levels in the intestinal fluid of offspring in the three groups at PD28 following maternal intervention with Clostridium butyricum. Following weaning, no statistically significant differences were detected between the intervention groups and the control group. All values are expressed as the mean \pm standard deviation. Aa, control group; Ba, maternal intervention group; $\mathrm{Ab}$, offspring intervention group; $\mathrm{Bb}$, maternal and offspring intervention group; PD, postnatal days; sIgA, secreted immunoglobulin A.

the gut microflora without the Clostridium butyrium population increasing (14). It may promote the accumulation and development of beneficial bacteria such as Lactobacillus spp. and Bifidobacterium spp. by producing low lactose.

A typical example for the association between intestinal flora and the development of the host's immune system is the study of germ-free animals in, which a distinct decrease of Peyer's patches and a reduction of sIgA were found (18). Once germ-free animals were exposed to bacteria constituting a normal intestinal flora, the development of immune system occurred. Above of all, the intestinal flora has an important influence on the normal immune system.

It is accepted that the gastrointestinal tract is the largest immune organ due to the existence of gut-associated lymphoid tissue, which has a crucial role in distinguishing potentially pathogenic microbiota and harmless antigens, and preventing the invasion of pathogens by intestinal epithelial cells and sIgA in the innate immune system $(19,20)$. Once the first line of defense fails, the adaptive immune system participates in the immune defense through cellular immunity and humoral immunity mediated by Th1 and Th2 cells (3). Th1 and Th2 cells are differentiated from the same precursor cell, the Th0 cell. In the process of balancing between Th1 and Th2 cells, cytokines have an irreplaceable role. For instance, IL-2, IL-12 and IFN- $\gamma$ promote the Th1 cell and inhibit Th2 cell formation, while IL-4 and IL-10 promote the Th2 cell and inhibit Th1 cell formation (21). Autoimmune and allergic diseases may occur under circumstances where this balance is disturbed. Furthermore, it has been indicated that an excessive Th1-associated immune response is linked with autoimmune diseases such as rheumatoid arthritis, multiple sclerosis and graft vs. host disease. An excessive Th2-associated immune response has been associated with allergic diseases such as asthma and atopic dermatitis (22). Various probiotics were demonstrated to have different roles in the host's immune system. For instance, Lactobacillus paracasei (strain KW3110) may potentially reduce allergy-induced inflammation by inhibiting Th2 cytokines (11).

The present study demonstrated that the intestinal $\operatorname{sig} \mathrm{A}$ content in mice offspring demonstrated no significant differences when the maternal mice or their offspring were supplemented with Clostridium butyrium during lactation, which implys that the supplement may not affect the levels of sIgA. Th1-associated cytokine activation was slightly increased in the two intervention groups, Clostridium butyrium had no significant effect on the secretion of cytokines. The capacity of Clostridium butyrium supplementation to improve sIgA and regulate the microbiotic flora in the intestine suggests that it may aid in preventing the imbalance of Th1/Th2 and helping to prevent the occurrence of autoimmune diseases. Clostridium butyrium did not have a significant effect on intestinal $\operatorname{sIgA}$ content and cytokine secretion, which implies that it may not directly disrupt the immune systems of mice. In conclusion, supplementation with Clostridium butyrium (CGMCC no. 0313-1) regulated the balance of the intestinal floral, manifesting in decreased colony counts of Enterococcus spp. and Enterobacterium spp., and increased colony counts of Lactobacillus spp. and Bifidobacterium spp. in neonatal mice. Clostridium butyrium may also enhance the capacity of intestinal resistance to infections by increasing the secretion of $\operatorname{sig} \mathrm{A}$. However, intervention with Clostridium butyricum did not significantly affect the balance of Th1/Th2. Overall, Clostridium butyricum 
supplementation may be a safe method for regulating the establisment of the intestinal flora and acquired immune system development and should be explored in greater depth.

\section{Acknowledgements}

The present study was financially supported by the Science and Technology Project of Sichuan Province of China for (no. 2012JY0008). The authors would like to thank the Campus Public Health Center of Sichuan University (Chengdu, China) for counting the microbial culture technology and helping with the data interpretation.

\section{References}

1. Schuijt TJ, van der Poll T, de Vos WM and Wiersinga WJ: The intestinal microbiota and host immune interactions in the critically ill. Trends Microbiol 21: 221-229, 2013.

2. Rodriguez JM, Murphy K, Stanton C, Ross RP, Kober OI, Juge N, Avershina E, Rudi K, Narbad A, Jenmalm MC, et al: The composition of the gut microbiota throughout life, with an emphasis on earlylife. Microb Ecol Health Dis 26: 26050, 2015.

3. Kidd P: Th1/Th2 balance: The hypothesis, its limitations, and implications for health and disease. Altern Med Rev 8: 223-246, 2003.

4. Fujiwara D, Inoue S, Wakabayashi H and Fujii T: The anti-allergic effects of lactic acid bacteria are strain dependent and mediated by effects on both Th1/Th2 cytokine expression and balance. Int Arch Allergy Immunol 135: 205-215, 2004.

5. Vandenplas Y: Healthy gut microbiota and long term health. Benef Microbes 6: 173-179, 2015.

6. Bustos Fernandez LM, Lasa JS and Man F: Intestinal microbiota: Its role in digestive diseases. J Clin Gastroenterol 48: 657-666, 2014.

7. Björkstén B, Naaber P, Sepp E and Mikelsaar M: The intestinal microflora in allergic Estonian and Swedish 2-year-old children. Clin Exp Allergy 29: 342-346, 1999.

8. Valsecchi C, Marseglia A, Montagna L, Tagliacarne SC, Elli M, Licari A, Marseglia GL and Castellazzi AM: Evaluation of the effects of a probiotic supplementation with respect to placebo on intestinal microflora and secretory IgA production, during antibiotic therapy, in children affected by recurrent airway infections and skin symptoms. J Biol Regul Homeost Agents 28: 117-124, 2014.

9. Edwards CA and Parrett AM: Intestinal flora during the first months of life: New perspectives. Br J Nutr 88 (Suppl 1): S11-S18, 2002.
10. Yoshimatsu Y, Yamada A, Furukawa R, Sono K, Osamura A, Nakamura K, Aoki H, Tsuda Y, Hosoe N, Takada N and Suzuki Y: Effectiveness of probiotic therapy for the prevention of relapse in patients with inactive ulcerative colitis. World J Gastroenterol 21: 5985-5994, 2015.

11. Tsai YT, Cheng PC and Pan TM: The immunomodulatory effects of lactic acid bacteria for improf oving immune functions and benefits. Appl Microbiol Biotechnol 96: 853-862, 2012.

12. Nakanishi S, Kataoka K, Kuwahara T and Ohnishi Y: Effects of high amylose maize starch and Clostridium butyricum on metabolism in colonic microbiota and formation of azoxymethane-induced aberrant crypt foci in the rat colon. Microbiol Immunol 47: 951-958, 2003.

13. Scheppach W: Effects of short chain fatty acids on gut morphology and function. Gut 35 (1 Suppl): S35-S38, 1994.

14. Ichikawa $H$, Kuroiwa $T$, Inagaki A, Shineha R, Nishihira $T$, Satomi S and Sakata T: Probiotic bacteria stimulate gut epithelial cell proliferation in rat. Dig Dis Sci 44: 2119-2123, 1999.

15. Seki H, Shiohara M, Matsumura T, Miyagawa N, Tanaka M, Komiyama A and Kurata S: Prevention of antibiotic-associated diarrhea in children by Clostridium butyricum MIYAIRI. Pediatr Int 45: 86-90, 2003

16. Küster T, Zumkehr B, Hermann C, Theurillat R, Thormann W, Gottstein B and Hemphill A: Voluntary ingestion of antiparasitic drugs emulsified in honey represents an alternative to gavage in mice. J Am Assoc Lab Anim Sci 51: 219-223, 2012

17. Mitsou EK, Kirtzalidou E, Oikonomou I, Liosis G and Kyriacou A: Fecal microflora of Greek healthy neonates. Anaerobe 14: 94-101, 2008.

18. Falk PG, Hooper LV, Midtvedt T and Gordon JI: Creating and maintaining the gastrointestinal ecosystem: What we know and need to know from gnotobiology. Microbiol Mol Biol Rev 62: 1157-1170, 1998.

19. Purchiaroni F, Tortora A, Gabrielli M, Bertucci F, Gigante G, Ianiro G, Ojetti V, Scarpellini E and Gasbarrini A: The role of intestinal microbiota and the immune system. Eur Rev Med Pharmacol Sci 17: 323-333, 2013.

20. Kaetzel CS: Cooperativity among secretory $\operatorname{Ig}$ A, the polymeric immunoglobulin receptor, and the gut microbiota promotes host-microbial mutualism. Immunol Lett 162: 10-21, 2014.

21. de Jong R, Brouwer M, Kuiper HM, Hooibrink B, Miedema F and van Lier RA: Maturation- and differentiation-dependent responsiveness of human CD4+ T helper subsets. J Immunol 149: 2795-2802, 1992.

22. Pessi T, Sütas Y, Hurme M and Isolauri E: Interleukin-10 generation in atopic children following oral Lactobacillus rhamnosus GG. Clin Exp Allergy 30: 1804-1808, 2000. 